\title{
LXIV. Experiments on gaseous interference
}

\section{William Charles Henry M.D. F.R.S.}

To cite this article: William Charles Henry M.D. F.R.S. (1836) LXIV. Experiments on gaseous interference, Philosophical Magazine Series 3, 9:55, 324-333, DOI: $10.1080 / 14786443608649009$

To link to this article: http://dx.doi.org/10.1080/14786443608649009

曲 Published online: 01 Jun 2009.

Submit your article to this journal $\sqsubset$

Џll Article views: 4

Q View related articles 두 
are examples, he calls passive, because they do not act on sulphuric acid; the other, of which Faraday's quadro-carburetted hydrogen and the oil obtained by himself, as well as Mr. Enderby's oil (if the two latter be not identical), are specimens, he calls active, as they act strongly on the same acid. I have mentioned his views here, in order to direct attention to the striking fact, established by my experiments, that $\mathrm{Mr}$. Enderby's oil, belonging to one of these classes, is, partially at least, converted by the action of sulphuric acid into a liquid belonging to the other series, and retaining the same composition.

Another circumstance well worthy of notice is the fact that several of the liquids in question yield uniform results as to composition, even when the portions analysed differ in density and volatility. This has been shown to occur likewise in the oils of turpentine and lemons, and seems to indicate the existence of an almost unlimited number of polymeric combinations of carbon and hydrogen.

When Mr. Enderby's liquid was first made known it was stated to be an excellent solvent for caoutchouc, which, from its great volatility, would have rendered it extremely valuable. But it is necessary to state, that $I$ have not yet seen one specimen of it which, under any circumstances, in my hands, possessed this property. Two gentlemen informed me that they had succeeded in dissolving caoutchouc by means of it; but when asked to repeat the experiment with the same liquid they both failed. If therefore this liquid be a solvent for caoutchouc, it must be under conditions with which I am not acquainted.

\section{Experiments on Gaseous Interference. By WiLLiam Charles Henry, M.D., F.R.S.*}

THE singular power exerted by certain gases, of suspending the action of finely divided platina on mixtures of oxygen and hydrogen, was first observed and announced by Dr. Turnert, shortly after Döbereiner's discovery. It was also about the same period noticed by Dr. Henry $\ddagger$ in the course of his researches on the application of platina to the analysis of complex gaseous mixtures. More recently Dr. Faraday has described similar interferences, affecting the

* Read before the Chemical Section of the British Association at Bristol: and communicated by the Author.

$\dagger$ Jameson's Journal, vol. xi. pp. 99 and 311.

$\ddagger$ Phil. Trans., 1824, p. 266.; [ or Phil. Mag., First Series, vol. lxr. p. $27 \mathcal{2}_{0}-$-EDrt.]

$\oint$ Ibid., 1834, p. Zl. 


\section{Dr.W.C. Henry's Experiments on Gaseous Interference. 325}

activity of prepared plates of the same metal. Various modes have been proposed of interpreting these phænomena. Dr. Turner (Chemistry, 5th edit., p. 647) observes, "One would be tempted to suppose that these gases act by soiling the metallic surface, though in some respects this explanation is not satisfactory." In the Essay by Dr. Henry already referred to (p. 282), it is suggested that this property " is most remarkable in those gases which possess the strongest attraction for oxygen, and it is probably to the degree of this attraction, rather than to any agency arising out of their relations to caloric, that we are to ascribe the various powers which the gases manifest in this respect." Finally, the latest writer on this subject, Dr. Faraday, concludes (par. 655.): "Whether the effect produced by such small quantities of certain gases depends upon any direct action which they may exert upon the particles of oxygen and hydrogen, by which the latter are rendered less inclined to combine, or whether it. depends upon their modifying the action of the plate temporarily (for they produce no real change on it) by investing it through the agency of a stronger attraction than that of the hydrogen, or otherwise, remains to be decided by more extended experiments." It was in the hope of illustrating the nature of these interesting phænomena in gaseous chemistry that the following experiments were instituted.

The carbonic oxide and olefiant gases are stated by all the above-mentioned inquirers to possess, in the most remarkable degree, the property of interference. I have selected those gases as fitter subjects for experiment than others which manifest the same property, because, as Dr. Faraday testifies, they prevent combination, without at all injuring or affecting the power of the platina (644. and 6.), and hence exhibit the phænomenon in its simplest form. The platina which $1 \mathrm{em}-$ ployed was in the various conditions of (1.) plates prepared according to Dr. Faraday's method; (605.) (2.) sponge from calcination of the ammonia-muriate, either alone or moulded with China clay into small balls; and (3.) the black powder precipitated by the addition of alcohol to the solution of protochloride of platina in potassa, according to Liebig's process.

I. Carbonic Oxide.-To a mixture of oxygen and hydrogen, in the proportions required to constitute water, carbonic oxide was added, so as to form $\frac{1}{12}$ th, $\frac{1}{19}$ th, and $\frac{1}{30}$ th of the entire volume. The prepared plate when admitted caused no appreciable diminution, nor was any change observed during three or four hours in any of the three mixtures. The following morning evident but slight diminution was visible in all. 


\section{Dr.W. C. Henry's Experiments on Gaseous Interference}

Since the gaseous products admit of exact analysis only over mercury, a fluid through which the plate cannot be passed without destroying the purity of surface essential to its activity, I was induced to employ balls of platina and China clay in the greater number of instances. Such balls, introduced over mercury into mixtures containing from $\frac{1}{2} t_{4}$ th to $\frac{1}{50}$ th of carbonic oxide, produced no instantaneous action. But in the course of five minutes there was generally a visible diminution of volume, and in the space of two hours the action was complete. When the carbonic oxide formed a larger element of the mixture, as from $\frac{1}{8}$ th to $\frac{1}{3}$ rd, the action was still longer delayed. With one third there was scarcely any visible decrease of volume during the first hour; and even on the following day there remained much gaseous matter unconsumed. Thus the residue of a mixture of $\cdot 35 \mathrm{cu}$. in. of carbonic oxide with 70 of hydrogen and oxygen in combining proportions (together $\mathbf{1} \cdot 05$ ), amounted after twentyfour hours' contact with the ball to 82 . The proportions were extensively varied between $\frac{1}{2}$ and $\frac{1}{3_{0}}$ th as limits; and between these it was observed, that combination to a greater or less amount was in all cases induced by a sufficient duration of contact. Carbonic oxide, it appears then, does not prevent but only retards gaseous union.

To ascertain the proportions in which the oxygen had been shared by the gases opposed to each other, the residue was washed with limewater or with caustic potassa. In all cases a notable diminution, due to the absorption of carbonic acid, was observed, and from the limewater carbonate of lime was precipitated. The quantity of carbonic acid so formed varied with the varying proportion which the carbonic oxide had borne to the hydrogen of the explosive mixture. When the hydrogen and carbonic oxide were present in equal volumes, and the oxygen was sufficient to saturate one of them only, I found that the carbonic oxide had appropriated to itself, in one experiment eight times, in another as much as ten times, the volume of oxygen that the hydrogen had taken. When the carbonic oxide was inferior in volume to the hydrogen, less oxygen was expended in the production of carbonic acid, but still considerably more in proportion than had combined with the hydrogen. It is then a general fact, that carbonic acid is generated in all cases in which carbonic oxide suspends the action of platina on mixtures of oxygen and hydrogen.

The next object of inquiry was to ascertain the action of platina in its various forms on mixtures of carbonic oxide with oxygen only. Dr. Faraday had noticed that at common temperatures "a mixture of two volumes carbonic oxide and 
one volume oxygen was unaffected by the prepared platina plate in several days." (57*.) I found however that after four days' contact with the plate, an appreciable diminution had ensued. 'Thus $3 \mathrm{cu}$. in. of such a mixture were reduced to $2 \frac{3}{4}$, indicating the formation of nearly $\frac{1}{2} \mathrm{cu}$. in. of carbonic acid. But the union of carbonic oxide with oxygen was much accelerated by causing the gases to stand in contact with the platina over a solution of caustic potassa, instead of distilled water, which had been used by Dr. Faraday. In such experiments there was a daily decrease of volume in the confined gases, till the fluid by ascending covered the greater part of the plate. Thus $3 \frac{1}{4} \mathrm{cu}$. in. were reduced to $1 \mathrm{cu}$. in. in about seven days.

Platina in the form of sponge had been previously stated to cause the slow union of carbonic oxide and oxygen*. This action $I$ found was also quickened by admitting potassa fusa with or without water above the confining surface of mercury. Thus $1.40 \mathrm{cu}$. in. of a mixture of carbonic oxide and oxygen in equivalent proportions became 1.25 in the first five minutes after introducing the platina sponge and potassa; in the next five minutes $1 \cdot 15$, and in half an hour much less than $1 \mathrm{cu}$. in. remained. In two hours less than half a cubic inch was lefit, and the potassa, by rising so as to moisten the sponge, terminated the experiment. Finally, the black pow, der of Liebig admitted into mixtures of carbonic oxide and oxygen became incandescent at the moment of contact, and continued to glow until all the carbonic oxide was converted into carbonic acid. Platina therefore, in all its forms, determines, at atmospheric temperatures, the union of carbonic oxide with oxygen. In the state of plate the change goes on with extreme slowness; in that of sponge, much more quickly; and in the most minutely divided condition of powder, with ignition and with great rapidity.

In this unequivocal action of platina upon mixtures of oxygen with carbonic oxide only, it appears to me, is to be found the interpretation of the property residing in the latter gas, of suspending the combining tendencies of hydrogen and oxygen in mixtures of the three gases. Possessing, as has been already shown, a stronger affinity for oxygen than is inherent in hydrogen, carbonic oxide seizes, when mingled with those two gases and exposed to the agency of platina, a much larger proportion of the oxygen than is due to its comparative volume. In such complex mixtures, just as in simple mixtures of carbonic oxide and oxygen, the slow formation of carbonic

* Phil. Trans., 1824, p. 267. [Phil. Mag., vol. lxv. p. 269.-Edix.] 
328 Dr.W.C. Henry's Experiments on Gaseous Interference.

acid continues to be the predominant action. Hence the phænomenon of "interference" is better explained by contemplating the gaseous mixture as one of carbonic oxide and oxygen to which hydrogen has been added, than as one of hydrogen and oxygen with which carbonic oxide has been mingled. Such a simple mixture of carbonic oxide and oxygen, in presence of the prepared plate or sponge, is in a state of slow acidification; and the admission of hydrogen, a gas endowed with a feebler affinity for oxygen, occasions no essential change of the chemical actions previously in operation.

This view of the mode in which carbonic oxide overrules the combining tendencies of hydrogen and oxygen is supported by experiments, long ago recorded, on the influence of increased temperature. For it has been shown* that the phænomena of interference disappear at a heat between $300^{\circ}$ and $340^{\circ}$ Fahrenheit, the union of the elements of a gaseous mixture which is slowly acted upon at common temperatures being then rapidly accomplished. Now this is precisely the temperature at which carbonic oxide, when heated simply with its equivalent of oxygen in presence of the platina sponge, is rapidly converted into carbonic acid. Instant action, with incandescence, $I$ have found also follows the admission of the black powder into mixtures of hydrogen, oxygen and carbonic oxide; and it has been already stated that simple mixtures of carbonic oxide and oxygen (or carbonic oxide alone, the oxygen being supplied by the powder, are inflamed by platina in the state of powder. The phænomena of interference are observed then only at those temperatures, and with that form of platina, which induce the slow union of carbonic oxide and oxygen; and wholly disappear at the higher temperature, or with that more active state of the metal which causes rapid combination.

An obvious objection, however, presents itself to the view that carbonic oxide possesses a stronger affinity for oxygen than hydrogen exerts, viz. that while bydrogen and oxygen are speedily detonated at common temperatures by the plate or sponge, the union of carbonic oxide and oxygen takes place with great slowness. The explanation of this apparent anomaly I believe to be, that the product of the combustion of hydrogen (aqueous vapour) at once quits the surface of the metal, and is liquefied by the cold sides of the tube; while the combustion of the carbonic oxide yields a gas which remains for a while adherent to the metallic surface next to which it is generated, and thereby prevents a sufficiently rapid

* Dr. Henry, Phil. Trans. 1824, pp. 278 and 280. 
access of fresh unaltered gas to elevate materially the temperature of the platina. In confirmation of this view I found that caustic potassa, by absorbing the carbonic acid as fast as it is formed, accelerates the acidification of carbonic oxide. When the metallic superficies is so extensive (for instance, in the powder of Liebig,) that a high temperature is quickly attained by the metal in contact with the first portions of gas that are consumed, it has been already shown that carbonic oxide, like hydrogen, then unites with oxygen, with incandescence. Finally, it is well known that even mixtures of hydrogen and oxygen do not detonate on first admitting the prepared plate. During the first minute the union is generally very slow, and it only becomes explosive when the temperature of the plate has been raised by its action upon the gaseous mixture.

2. Olefiant Gas.-The olefiant gas which I employed had been carefully washed with caustic potassa, and in the experiments made over mercury, it had stood for many days in contact with dry potassa fusa. It resulted from all my experiments with platina in its various conditions, that the property of interference is much less energetic in olefiant gas than in carbonic oxide*. Of this I could not feel satisfied without repeated examination, because the reverse is the result of Dr. Faraday's experience, according to which olefiant gas interferes when it constitutes $\frac{1}{4} \frac{1}{8}$ th of the entire mixture, and carbonic oxide only when it amounts to $\frac{1}{8}$ th. I found, however, that in a mixture of $3.00 \mathrm{cu}$. in. explosive mixture with $\cdot 08$ of olefiant, in which the olefiant is about $\frac{1}{5} \frac{1}{8}$ th of the whole, the plate began to act visibly on first admission. In ten minutes there remained $2 \cdot 50$, and in a quarter of an hour 2.00 , when the action became very rapid, the plate being so hot as to cause the ascending water, in contact with it, to boil, and only $.25 \mathrm{cu}$. in. were left unconsumed. Even when the olefiant amounted to $\frac{1}{19}$ th of the entire mixture there was manifest action in the course of a quarter of an hour, and in two days the water had covered the plate.

Olefiant gas in much larger proportion, even when constituting $\frac{1}{4}$ th or $\frac{1}{5}$ rd of the mixture, did not in the slightest degree retard the action of the platina balls or sponge. Several trials were made, in which the olefiant and explosive mixture were mingled in equal proportions. In all of these the ball acted instantaneously, and ascended rapidly into the tube during one or two minutes, when its rise was suddenly checked. Thus $1.02 \mathrm{cu}$. in. were reduced in the first minute to 90 , and became $\cdot 82$ after an hour's contact. The following day only $\cdot 56$

- See also Mr. Graham's Experiments, Journal of Science, 1829, p. 356. Third Series. Vol.9. No. 55. Nov. 1836. $2 \mathrm{Q}$ 
were left, which were not diminished by washing with potassa. Even when the volume of the olefiant was double that of the explosive mixture, there was instant action, though to a less extent than in the last experiment.

When the volume of the olefint was three times that of the explosive mixture, no immediate action was visible, though a notable diminution always took place in the course of a few hours or on the following day. Finally, the activity of the black powder of Liebig was not suspended by the addition of twenty volumes of olefiant to one of explosive mixture.

On subjecting to examination the gaseous products of these and other experiments on olefiant gas, mingled with various proportions of the explosive mixture, very different results from those afforded with carbonic oxide were obtained. In the greater number of experiments with olefiant gas, no appreciable diminution was caused by prolonged contact, or by subsequent washing with potassa; and though in some cases, when the explosive mixture considerably exceeded the olefiant, there was a perceptible absorption, yet the carbonic acid thus evidenced was always of small amount. In three successive experiments, in which olefiant gas and explosive mixture were mingled in equal proportions, there was no measurable product of carbonic acid. Yet in all these cases, though the ball ascended rapidly on first contact, its activity was soon suspended. Olefiant gas then possesses, like carbonic oxide, an undoubted power of retarding the union of hydrogen and oxygen, but it differs from carbonic oxide in not necessarily affording carbonic acid by so acting.

Similar differences presented themselves on submitting mixtures of olefiant and oxygen only to the action of platina. To the observation of Dr. Faraday, "that the most prolonged contact with the prepared plates never induced the union of the elements of olefiant with oxygen," I may add, that the contact was not efficient, even when aided by the presence of liquid potassa. The spongy metal, similarly conjoined with potassa, was also for the most part entirely inert, and in the few instances in which carbonic acid appeared, it was formed very slowly, and in small quantity. At the temperature $480^{\circ}$ Fahrenheit, however, the sponge has been shown to occasion the speedy though inexplosive combustion of olefiant in mixture with oxygen. Liebig's powder too caused the slow combination of the two gases at atmospheric temperatures, as was evidenced both by considerable diminution of volume, and by the test of lime-water; and when the tube containing the gaseous mixture and powder was surrounded with boiling water combination ensued with rapidity. Now the mode in which platina acts is of course identical in the various states 
of plate, sponge, or powder; the powder presenting only an infinitely larger extent of surface for gaseous contact than the plate or sponge. Hence the tendencies of the constituents of olefiant to unite with oxygen, which are evidenced by combination to considerable amount in presence of the powder, may be inferred to be operative, though less effectively, on the surface of the same metal under other forms; and viewed in connexion with the unequivocal proofs of the nature of the interference of carbonic oxide, these tendencies may be admitted to furnish an adequate explanation of the more feeble interference of olefiant gas*.

The influence of temperature in quickening the action of the sponge or powder on mixtures of olefiant and oxygen strongly confirms this view, when taken in connexion with a peculiarity observed in the mode of interference of olefiant gas. Thus it has been stated that olefiant mingled in equal bulk with explosive mixture did not prevent instant action; and that it began to interfere only when much of the oxygen and hydrogen had combined together, with the disengagement of great heat. The interfering power of olefiant gas, feeble at atmospheric temperatures, is then greatly augmented by heat, which has been already shown to determine the separate combination of the elements of olefiant with oxygen, and which is well known to exalt chemical affinity.

In recapitulation, it may be stated that carbonic oxide interferes with the action of platina upon mixtures of oxygen and hydrogen, by virtue of its stronger affinity for oxygen, which causes it slowly to take the larger portion of that gas. Olefiant gas, which at common temperatures has a weaker attraction for oxygen than hydrogen, suspends the combining tendencies of those two gases, only when its volume greatly exceeds that of the mixture, in which case the weaker affinity is aided by a greater number of atoms. Even with this advantage olefiant is unable to appropriate the oxygen to itself, but only retards its union with hydrogen by opposing a weaker attractive tendency.

Nor is the admission of attractive forces between the particles of mixed gases, even when not manifested by any visible action (as between oxygen and the elements of olefiant gas), inconsistent with what is known of chemical affinity as operating in the solid and liquid forms of matter. In the eighth of his admirable series of memoirs on electro-chemistry, Dr. Faraday has shown that zinc, having its surface thinly amal-

- The interfering power of carbonic oxide as respects the action of the platina balls is eighteen times as great as that of olefiant; carbonic oxide in the proportion of $\frac{1}{2}$ th interfering as completely as olefiant in that of $\frac{3}{4}$ ths of the mixture. 


\section{Dr.W.C. Henry's Experiments on Gaseous Interference.}

gamated, though incapable, when immersed in dilute sulphuric acid, of effecting the decomposition of water, has yet the power, by its attraction for the oxygen of the particles of water in contact with its surface, to induce a peculiar state of electrical tension or polarity in those particles of water, as well as a similar but opposite state in the contiguous particles of zinc. By plunging a platina plate into the solution and completing the galvanic circle, this tension is relieved and decomposition of water instantly ensues. In the preparatory stage of this important experiment we have then a case of chemical forces in undoubted operation, yet giving no appreciable sign of their existence.

Other arguments present themselves in favour of the opinion, that the property inherent in certain gases of preventing or retarding the union of hydrogen and oxygen is to be referred to their attraction for oxygen, and not to any peculiar action of the metallic surface, by which it becomes invested with the interfering gas. (1.) All the gases which have been hitherto observed to exhibit this power, are such as are capable of uniting with oxygen; and the non-interfering gases are such as cannot, at least within a considerable range of temperature, be brought to combine with that element. (2.) The property of interference follows, in its comparative energy, the same order as the respective combustibilities of the gases. Thus Sir Humphry Davy observed that carbonic oxide and olefiant were most inflammable, while carburetted hydrogen required for its combustion a much higher temperature, being neither fired by white-hot charcoal nor iron. Dr. Henry also has shown that in presence of the sponge, carbonic oxide combines rapidly with oxygen at from $300^{\circ}$ to $340^{\circ}$ Fahrenheit; olefiant at $520^{\circ}$; and carburetted hydrogen not at any temperature to which the mercurial bath could be raised. I have ascertained that the two first gases observe the same order of union in presence of Liebig's powder, carbonic oxide inflaming at atmospheric temperatures, and olefiant gas being rapidly acted upon at $212^{\circ}$. Now this progression is precisely that of their interfering powers, carbonic oxide acting when it constitutes only $\frac{1}{2} 4$ th of the mixture, olefiant gas not suspending action till it amounts to $\frac{3}{4}$ ths, and carburetted hydrogen possessing no power whatever of retarding the action of platina even when its volume exceeds by ten times that of the explosive mixture. Finally, that the restraining force is wholly dependent upon the relations of the mixed gases among one another, is deducible from the fact that the same gases which suspend the combining tendencies of hydrogen and oxygen in presence of platina, resist also other modes of effectuating the union of those gases; 
for instance, the discharge of a Leyden jar. The curious facts observed by Professor Graham, respecting the power of even smaller quantities of the same and other gases to arrest the slow oxidation of phosphorus, are also favourable to the doctrine that the interfering gases act solely by virtue of their superior attractions for oxygen.

LXV. Simple Method of proving the Law of Gravitation. By J. R. Young, Esq., Professor of Mathematics in Belfast College. ${ }^{*}$

THE following is a concise mode of establishing the law of gravitation, with the aid of only the most obvious dynamical principles. Its novelty, and remarkable simplicity, may perhaps entitle it to a place in the Philosophical Magazine.

Let $\mathbf{R}$ be the radius of curvature at any point of the planetary orbit, $r$ the distance of the planet from the sun, $p$ the semiparameter, and $\mathbf{P}$ the perpendicular from the centre of force upon the tangent through the extremity of $r$.

By a known property of the conic sections, if from the foot of the normal, $\mathrm{N}$, a perpendicular be drawn to the radius vector, the part intercepted between this perpendicular and the curve will be equal to the semiparameter. Hence by similar triangles we shall have

$$
\frac{r}{\mathrm{P}}=\frac{\mathrm{N}}{p} \text {. }
$$

Now from the usual expression for the radius of curvature we have

$$
\mathrm{R}=\frac{\mathrm{N}^{3}}{p^{2}}=\mathrm{N} \frac{r^{2}}{\mathrm{P}^{2}}
$$

Moreover, by the resolution of forces,

$$
\frac{\mathrm{N}}{p}\left(=\frac{r}{\mathrm{P}}\right)=\frac{\text { force of gravitation }}{\text { normal force }}
$$

But from the well-known theorem of Huygens, the normal force is expressed by $\frac{v^{8}}{\mathrm{R}}$; and it is an obvious deduction from the first law of Kepler that the velocity varies inversely as the perpendicular upon the tangent. (Principia, sect. 2. prop.1. cor. 1.) Hence

$$
\text { Normal force }=\frac{v^{2}}{\mathbf{R}}=\frac{\mathbf{C}^{2}}{\mathrm{P}^{2} \overline{\mathrm{R}}}=\frac{\mathbf{C}^{3}}{\mathbf{N} r^{2}}
$$

Consequently (1.)

* Communicated by the Author. 